Société d'histoire de la révolution de 1848 et des

révolutions du XIXe siècle

$59 \mid 2019$

Souverainetés africaines

\title{
Anatole LE BRAS, Un enfant à l'asile. Vie de Paul Taesch (1874-1914), préface de Philippe Artières
}

\section{Hervé Guillemain}

\section{OpenEdition \\ Journals}

Édition électronique

URL : https://journals.openedition.org/rh19/6655

ISSN : 1777-5329

Éditeur

La Société de 1848

Édition imprimée

Date de publication : 30 décembre 2019

Pagination : 223-225

ISSN : 1265-1354

Référence électronique

Hervé Guillemain, «Anatole LE BRAS, Un enfant à l'asile. Vie de Paul Taesch (1874-1914), préface de Philippe Artières », Revue d'histoire du XIXe siècle [En ligne], 59 | 2019, mis en ligne le 11 janvier 2020, consulté le 11 janvier 2023. URL : http://journals.openedition.org/rh19/6655

Ce document a été généré automatiquement le 11 janvier 2023.

Tous droits réservés 


\title{
Anatole LE BRAS, Un enfant à l'asile. Vie de Paul Taesch (1874-1914), préface de Philippe Artières
}

\author{
Hervé Guillemain
}

\section{RÉFÉRENCE}

Anatole LE BRAS, Un enfant à l'asile. Vie de Paul Taesch (1874-1914), préface de Philippe Artières, Paris, CNRS Éditions, 2018, 297 p., 22 euros.

1 Les travaux universitaires français ayant pour objet l'histoire de la folie et de la psychiatrie aux $\mathrm{xIX}^{e}$ et $\mathrm{xx}^{\mathrm{e}}$ siècles présentent ces dernières années plusieurs caractéristiques communes. En premier lieu, ils développent une approche résolument sociale qui achève de désenclaver et de « dépsychiatriser » l'histoire de la psychiatrie ${ }^{1}$, confirmant en cela l'ancrage des historiens français de la santé dans le champ de l'histoire sociale. En second lieu, elle participe du « patient turn » que l'historien anglais Roy Porter appelait de ses vœux en s'appuyant sur l'idéal d'une histoire "from below ", promue notamment par Edward P. Thompson ${ }^{2}$. Enfin, elle s'est investie dans l'exploration massive des dossiers de patients qui constituent une mine pour l'historien du social. Le livre d'Anatole Le Bras participe de cette nouvelle histoire de la folie et de la psychiatrie.

2 L'époque dont il est question dans le livre, et qui correspond aux dates du personnage central du récit Paul Taesch (1874-1914), n'est plus un angle mort de l'historiographie de la psychiatrie. Alors que de nombreux travaux avaient été consacrés à la reconstitution de l'émergence du système psychiatrique français dans les deux premiers tiers $d u$ xix ${ }^{e}$ siècle et que d'autres prenaient en considération la crise de ce même système lors des deux guerres mondiales, la période 1870-1914 a longtemps été considérée comme un temps faible de l'histoire de la psychiatrie. Elle a ainsi souvent été réduite à une période d'encombrement démographique des asiles, d'impasse 
thérapeutique de la médecine aliéniste, ainsi qu'à la volonté politique de réformer la loi de 1838 régissant les modes d'internement des patients. Or plusieurs historien.ne.s, au premier chef desquels Aude Fauvel et plus récemment Marie Derrien, ont montré combien cette période était importante pour comprendre les débats et les évolutions du premier $\mathrm{Xx}^{\mathrm{e}}$ siècle ${ }^{3}$.

Dans cet ouvrage, Anatole Le Bras montre notamment que la période est marquée par un nouvel intérêt pour les maladies mentales de l'enfance, jusqu'alors peu considérées - les premiers grands travaux médicaux sur la folie des enfants datant des années 1860-1870. Des services spécialisés dans la prise en charge des enfants « idiots » ont fait leur apparition dans les hôpitaux et les asiles de France entre les années 1880 et 1910, parmi lesquels le plus célèbre est celui du Docteur Bourneville, médecin républicain radical et anticlérical promoteur d'une nouvelle approche médico-éducative.

4 Le récit de l'auteur, qui évoque donc l'histoire d'un enfant à l'asile, se situe durant cette période. Son originalité réside dans la manière dont l'historien cherche à suivre son sujet à travers les méandres institutionnels de la psychiatrie et de l'assistance. Car s'il est facile de travailler sur la biographie d'un individu à partir d'un dossier de patient particulièrement riche, il est plus ardu de retracer le parcours trans-institutionnel de ce même individu. Le Bras débute son enquête par une trouvaille, celle des mémoires de Paul Taesch, jeune homme interné à l'âge de douze ans et pensionnaire des asiles de Bicêtre, Ville Evrard et Quimper, un texte minutieusement reproduit - ainsi que l'ensemble des pièces d'archives afférentes à la vie de Paul Taesch-dans les cent premières pages du livre. Les parties qui suivent présentent les cadres scientifiques qui régissent la psychiatrie infantile de l'époque, la vie du sujet dans les différentes institutions dans lesquelles il est interné, le parcours de l'individu en dehors de l'assistance asilaire.

5 Ce travail est un tour de force car il donne naissance à un portrait très incarné du personnage. Paul Taesch est un orphelin qui n'a pratiquement connu que l'univers institutionnel, celui des orphelinats avant celui des asiles. Il entre en relation avec ses frères et sœurs au parloir de l'asile. Le parcours n'est en soi pas extraordinaire, car les établissements psychiatriques sont de longue date un lieu de relégation pour nombre de jeunes placés à l'assistance publique. Dans ses mémoires, Taesch explique son évolution "vicieuse" par les mauvais traitements reçus dans les institutions qui l'accueillirent dans son enfance. Mais le jeune homme est surtout façonné par l'asile. Comme certains furent considérés et qualifiés comme de "bons malades" asilaires, Paul Taesch est assurément un " mauvais malade ", « évadeur » récidiviste, simulateur, contestataire, rebelle, ballotté d'une institution à l'autre, connu défavorablement des services d'assistance sanitaires et sociaux, assimilé à un parasite. L'attitude des institutions psychiatriques vis-à-vis de cet individu peut apparaître ambiguë : elle ne veut plus de ces personnes dont elle a créé l'incapacité à vivre au dehors et leur permet souvent de vivre de longues années à l'abri du besoin. On est loin du grand récit de l'enfermement des fous tel que Michel Foucault a pu l'établir dès les années 1960 et qui subordonne l'institution asilaire à sa vocation répressive.

6 Un autre intérêt du livre est de donner toute sa place à la stratégie individuelle de Paul Taesch. Le jeune homme est loin d'être une victime. Assisté dans tous les sens du terme, il joue néanmoins avec le savoir de son temps en simulant les symptômes hystéroépileptiques. La stratégie simulatrice étant assez courante, elle suscite chez les psychiatres le désir de construire un savoir indépendant du récit du sujet et qui soit 
ancré sur la recherche des marqueurs biologiques de la maladie : c'est toute l'histoire à venir de la psychiatrie du $\mathrm{xx}^{\mathrm{e}}$ siècle. On aimerait tout de même savoir dans quel objectif réel le mémoire de Taesch est écrit et quel effet cette visée peut produire sur le texte reproduit dans le livre. Car à l'évidence dans cette confession à la direction d'un asile, en faisant l'autoportrait d'un nuisible irresponsable qui va s'amender, Taesch reproduit des récits d'aveux plutôt courants, des récits qu'attendent les directeurs d'asiles comme de prisons pour justifier la sortie des enfermés.

7 Que devient le jeune adulte après sa sortie de l'asile en 1900 et jusqu'à son décès en 1914 ? Les traces sont ténues dans les archives d'un système d'assistance qui bricole des solutions de postcure et échoue assez largement à remettre les patients asilaires au travail et en société. Peut-être que les lecteurs de cette belle enquête microhistorique pourront donner quelques nouvelles de cet enfant d'asile devenu grand.

\section{NOTES}

1. Isabelle von Bueltzingsloexen, "Vers un désenclavement de l'histoire de la psychiatrie ", Le mouvement social, $\mathrm{n}^{\circ} 253,2015 / 4$, p. 3-11.

2. Alexandra Bacopoulos-Viau et Aude Fauvel, “The Patient's Turn Roy Porter and Psychiatry's Tales, Thirty Years On", Medical history, vol. 60, 2016/1, p. 1-18.

3. Voir leurs articles in: Alexandre Klein, Hervé Guillemain et Marie Claude Thifault, La fin de l'asile? Histoire de la déshospitalisation psychiatrique dans l'espace francophone au $\mathrm{Xx}^{e}$ siècle, Rennes, Presses universitaires de Rennes, 2018. 